ISSN: 2386-3919 - e-ISSN: 2386-3927

DOI: https://doi.org/10.14201/et2020382113133

\title{
FORMACIÓN INICIAL DEL PROFESORADO BRASILEÑO EN PREVENCIÓN DEL RIESGO SOCIAL SEGÚN LA LEGISLACIÓN EDUCATIVA
}

\author{
Initial Training of Brazilian Teachers in Prevention \\ of Social Risk According to Educational Legislation
}

\author{
Magda Maria Ventura Gomes DA Silva \\ Universidad Nacional de Educación a Distancia (UNED) \\ magdaventura@uol.com.br
}

Recibido: 09/12/2020; Aceptado: 18/12/2020; Publicado: 27/12/2020

Ref. Bibl. MAGDA MARIA VENTURA GOMES DA SILVA. Formación inicial del profesorado brasileño en prevención del riesgo social según la legislación educativa. Enseñanza E Teaching, 38, 2-2020, 113-133.

RESUMEN: Este artículo presenta los resultados del análisis de contenido realizado, a través del software Atlas.ti, a la legislación que regula la formación inicial del profesorado brasileño. En Brasil se accede a la carrera docente desde la Licenciatura en Pedagogía y desde el resto de las licenciaturas. Esta doble vía de acceso a la profesión docente obliga a analizar seis documentos legislativos. Los documentos analizados van desde las dos últimas leyes de educación vigentes en Brasil -Ley de Directrices y Bases de la Educación Nacional (Ley n. ${ }^{\circ}$ 9.394, 1996) y la Ley que aprueba el Plan Nacional de Educación (Ley n. ${ }^{\circ}$ 13.005, 2014)- hasta las cuatro Directrices Curriculares Nacionales. Las directrices curriculares nacionales, en Brasil, rigen la formación del profesorado en los cursos de Pedagogía (Parecer CNE/CP n. ${ }^{\circ}$ 5, 2005; Resolución, CNE/CP n. ${ }^{\circ}$ 1, 2006), así como en el resto de las licenciaturas (Parecer CNE/CP n. ${ }^{\circ}$ 2, 2015; Resolución CNE/CP n. ${ }^{\circ}$ 2, 2015). El objetivo de este artículo descriptivo de análisis documental del contenido de la legislación educativa vigente consiste en encontrar, tanto implícita como explícitamente, la capacitación ofrecida a los profesores en formación, sobre un aspecto primordial en la sociedad brasileña: la prevención del riesgo social. Las conclusiones de esta investigación 
MAGDA MARIA VENTURA GOMES DA SILVA

FORMACIÓN INICIAL DEL PROFESORADO BRASILEÑO EN PREVENCIÓN DEL RIESGO SOCIAL SEGÚN LA LEGISLACIÓN EDUCATIVA

cualitativa permiten afirmar dos hechos. Por un lado, se indica que en la legislación analizada existe una preocupación explícita desigual (dependiendo del documento analizado) por reducir manifestaciones visibles de riesgo social cristalizado. Por otro lado, se afirma que no existe referencia explícita a la formación docente para poder prevenir, desde la escuela, el riesgo social en las nuevas generaciones. Ante esta contradicción entre idea teórica y realización práctica se concluye afirmando que la prevención del riesgo social no debe considerarse una competencia docente en la formación del profesorado novel brasileño.

Palabras clave: formación de profesores; leyes sobre educación; prevención; riesgo social.

SUMMARY: This article presents the results of the content analysis carried out, through the Atlas.ti software, to the legislation that regulates the initial training of Brazilian teachers. In Brazil, the teaching career is accessed from the teacher training of Pedagogy and from the rest of the teacher training. This dual access path to the teaching profession requires the analysis of six legislative documents. The documents analyzed range from the last two education laws in force in Brazil -Law of Guidelines and Bases of National Education (Law n. ${ }^{\circ}$ 9.394, 1996) and the Law that approves the National Education Plan (Law n. ${ }^{\circ} 13.005$, 2014) - up to the four National Curricular Guidelines. The National Curricular Guidelines, in Brazil, govern the training of teachers in Pedagogy courses ("Parecer» CNE/CP n. ${ }^{\circ}$ 5, 2005; Resolution, $\mathrm{CNE} / \mathrm{CP}$ n. ${ }^{\circ} 1,2006$ ) as well as in the rest of the teacher training ("Parecer» $\mathrm{CNE} / \mathrm{CP}$ n. ${ }^{\circ}$ 2, 2015; Resolution CNE/CP n. ${ }^{\circ} 2$, 2015). The objective of this descriptive article of documentary analysis of the content of current educational legislation is to find, both implicitly or explicitly, the training offered to teachers in training, on a fundamental aspect in Brazilian society: the prevention of social risk. The conclusions of this qualitative research allow us to affirm two facts. On the one hand, it is indicated that in the legislation analyzed there is an unequal explicit concern (depending on the document analyzed) to reduce visible manifestations of crystallized social risk. On the other hand, it is stated that there is no explicit reference to teacher training to prevent, from school, social risk in new generations. Faced with this contradiction between theoretical idea and practical realization, it is concluded by stating that the prevention of social risk should not be considered a teaching competence in the training of new Brazilian teachers.

Key words: Teacher training; laws on education; prevention; social risk.

\section{INTRODUCCIÓN}

Brasil, con 211.755.692 habitantes, es uno de los países más poblados de América Latina (IBGE, 2020) y uno de los más violentos: «En los últimos años, la violencia en Brasil se ha incrementado exponencialmente» (Sampó, 2018). Además de un país violento, Brasil es el noveno país más desigual del mundo (IBGE, 2019). 
MAGDA MARIA VENTURA GOMES DA SILVA

FORMACIÓN INICIAL DEL PROFESORADO BRASILEÑO EN PREVENCIÓN DEL RIESGO SOCIAL SEGÚN LA LEGISLACIÓN EDUCATIVA

La desigualdad social y económica sobre la que se estructura el país desencadena múltiples manifestaciones de riesgo social.

El riesgo social es una realidad sociológica carente de definición unívoca (Rodríguez, 2013; Janczura, 2012; Quicios y Triguero, 2012). Esa falta de unicidad conlleva que el riesgo social suela describirse por las características que le son propias. Así, algunas de las características que lo definen serían que el riesgo social es una situación indeseada. Una circunstancia exógena a la persona que lo sufre. Un hecho que se desencadena por un conjunto de déficits que originan una serie nada desdeñable de efectos dañinos para la persona que lo padece (Quicios, 2007). El riesgo social se desencadena por "la inadecuada satisfacción de las necesidades y/o derechos» (Rodríguez, 2013, p. 15) de la persona. El riesgo social también se describe como la situación que impide a la persona en ella atrapada ejercer y disfrutar de sus derechos sociales como ciudadana.

Partiendo de todas las características que encierra esta lacra social, en este artículo se define el riesgo social como la situación en la que se encuentra una determinada persona debido a una serie de déficits relacionados con la familia de pertenencia, el entorno económico y sociocultural al que pertenezca, su salud física, psíquica y emocional-afectiva, entre otros factores desencadenantes que le impiden cubrir sus necesidades básicas, disfrutar de los derechos sociales inherentes a todos los ciudadanos o ambos aspectos a la vez (Santos, Roesch y Cruz, 2014; Quicios y Velloso, 2012; Lebrero y Quicios, 2011; Lorence, 2008; Almeida, Lisboa y Caurcel, 2007).

En definitiva, el riesgo social es la cristalización de realidades diversas, indeseadas y de efectos negativos sobre el sujeto que las padece estratificadas en intensidad. Esta amplitud de factores desencadenantes y estadios de intensidad justifican que el riesgo social en Brasil sea un fenómeno plural con múltiples dimensiones y diferentes grados. Sus manifestaciones visibles toman la forma de violencia sexual, agresiones, asaltos, tráfico de drogas, enfrentamientos con la policía, delincuencia, discriminaciones, violencia en las escuelas y en las comunidades, violación de los derechos humanos.

Todas estas manifestaciones de riesgo social son visibles para las personas que las sufren, pero, incomprensiblemente, resultan invisibles para el Estado.

En Brasil, existen innumerables problemas de:

- saneamiento y ausencia de servicios básicos (Gonçalves, 2013),

- trabajo infantil,

- uso de drogas,

- niños y adolescentes en las calles,

- embarazo precoz,

- discriminaciones (Rizzini y Brasil do Couto, 2019),

- asentamientos insalubres en favelas. 
Estas realidades se agravan, por dos motivos, por un lado, por la falta de información y, por otro, por las carencias para organizar acciones de prevención de los problemas (Schwonke, Fonseca y Gomes, 2009).

La compleja situación descrita afecta a las familias y a la sociedad, a las escuelas, a los alumnos y al profesorado. El profesorado no se siente ni informado ni preparado para poder abordar la prevención del riesgo social desde las escuelas.

Este artículo se centra, justamente, en los profesores y en su formación inicial para poder prevenir el riesgo social desde su actuación docente. Así, para conocer su formación inicial se analiza la legislación educativa que la regula.

En Brasil, los profesores cursan la Carrera de Licenciatura. Una vez concluida se obtiene el título de licenciado por dos vías. Una vía es la Licenciatura en Pedagogía. Otra vía es la obtención de licenciaturas diversas como Historia, Matemáticas, Ciencias, etc. El pedagogo, es decir, el licenciado en Pedagogía, ejercerá su docencia en la Educación Básica (niveles de educación infantil y primer tramo de la enseñanza fundamental). Los licenciados de las diferentes licenciaturas ejercerán su docencia en el segundo tramo de la enseñanza fundamental y en la secundaria.

Con independencia de la vía por la que se accede a la carrera docente, en todas las titulaciones el Ministerio de Educación tiene el mismo objetivo: formar a los docentes en competencias que les capaciten en la adquisición de conocimientos pedagógicos y desarrollo profesional. Para conseguir ese objetivo se articulan los diferentes planes de estudio. Así,

- Para la formación en Pedagogía, la estructura de los cursos se compone de tres núcleos y los planes de estudio se elaboran a partir de ellos (Resolución CNE/CP n. $.^{\circ} 1,2006$, art. $6^{\circ} .^{\circ}$.

- Para las demás licenciaturas, la estructura de los cursos también comprende tres núcleos con los planes de estudio derivados de ellos (Resolución CNE/CP n. ${ }^{\circ}$ 2, 2015, art. 12).

En el Cuadro 1 se relacionan los documentos legislativos analizados sobre la formación inicial del profesorado brasileño.

La primera ley analizada ha sido la Ley n. ${ }^{\circ}$ 9.394, del 20 de diciembre de 1996 (LDB). Esta ley establece las directrices y bases de la educación nacional brasileña. Esta ley se actualiza a través de otras. Una de ellas es la Ley n. ${ }^{\circ} 13.415$, del 16 de febrero de 2017, que establece las orientaciones sobre la Base Nacional Común Curricular (BNCC), además de otras determinaciones.

Según la Ley n. ${ }^{\circ}$ 9.394, del 20 de diciembre de 1996 (LDB), la educación comprende los procesos de formación desarrollados en la familia, convivencia humana, trabajo, instituciones de enseñanza e investigación, movimientos sociales y organizaciones de la sociedad civil y en las manifestaciones culturales (Ley n. ${ }^{\circ}$ 9.394, 1996, Título I, art. 1. ${ }^{\circ}$ ). Esta ley tiene sus luces y sombras. Si bien es cierto que ha propiciado importantes avances en la formación del profesorado (Pereira y Minasi, 2014; Santos, 2007), no ha cumplido con las expectativas que se tenían depositadas en la formación de los profesores en Brasil (Saviani, 2009). 
MAGDA MARIA VENTURA GOMES DA SILVA

FORMACIÓN INICIAL DEL PROFESORADO BRASILEÑO EN PREVENCIÓN DEL RIESGO SOCIAL SEGÚN LA LEGISLACIÓN EDUCATIVA

CUADRO 1

Identificación de los documentos legislativos analizados sobre formación inicial del profesorado brasileño

\begin{tabular}{|c|c|}
\hline $\begin{array}{l}\text { Ley n. }{ }^{\circ} 9.394 \text {, del } 20 \\
\text { de diciembre de } 1996\end{array}$ & $\begin{array}{l}\text { Establece las Directrices y Bases de la Educación Nacional - } \\
\text { LDB }\end{array}$ \\
\hline $\begin{array}{l}\text { Ley n. }{ }^{\circ} 13.005 \text {, del } \\
25 \text { de junio de } 2014\end{array}$ & Aprueba el Plan Nacional de Educación - PNE \\
\hline $\begin{array}{l}\text { Parecer } \mathrm{CNE} / \mathrm{CP} \\
\text { n. }{ }^{\circ} \text {, del } 13 \text { de } \\
\text { diciembre de } 2005\end{array}$ & $\begin{array}{l}\text { Establece las Directrices Curriculares Nacionales para el } \\
\text { Curso de Graduación en Pedagogía, licenciatura. Presenta la } \\
\text { fundamentación para el Curso de Pedagogía. Este Parecer fue } \\
\text { reexaminado por el Parecer CNE/CP n. }{ }^{\circ} 3 \text {, del } 21 \text { de febrero } \\
\text { de } 2006 .\end{array}$ \\
\hline $\begin{array}{l}\text { Resolución CNE/ } \\
\text { CP n. }{ }^{\circ} 1 \text {, del } 15 \text { de } \\
\text { mayo de } 2006\end{array}$ & $\begin{array}{l}\text { Establece las Directrices Curriculares Nacionales para el Curso } \\
\text { de Graduación en Pedagogía, licenciatura. }\end{array}$ \\
\hline $\begin{array}{l}\text { Parecer CNE/CP n. }{ }^{\circ} 2 \text {, } \\
\text { del } 9 \text { de junio de } 2015\end{array}$ & $\begin{array}{l}\text { Directrices Curriculares Nacionales para la Formación Inicial y } \\
\text { Continua de los Profesionales del Magisterio de la Educación } \\
\text { Básica. }\end{array}$ \\
\hline $\begin{array}{l}\text { Resolución CNE/ } \\
\text { CP n. }^{\circ} 2 \text {, del } 2 \text { de } \\
\text { julio de } 2015\end{array}$ & $\begin{array}{l}\text { Define las Directrices Curriculares Nacionales para la formación } \\
\text { inicial en nivel superior (cursos de licenciaturas, cursos de } \\
\text { formación pedagógica para graduados y cursos de segunda } \\
\text { licenciatura) y para la formación continua. }\end{array}$ \\
\hline
\end{tabular}

Fuente: Elaboración propia.

La segunda ley educativa analizada ha sido la Ley n. ${ }^{\circ}$ 13.005, del 25 de junio de 2014, que aprobó el Plan Nacional de Educación (PNE). Esta ley tiene una vigencia de diez años. Para cumplir lo que determina el artículo 214 de la Constitución brasileña, el Plan Nacional de Educación (PNE) debería articular el sistema de enseñanza nacional de educación en régimen de colaboración y definir las directrices, objetivos, metas y estrategias de implementación para asegurar el mantenimiento y desarrollo de la enseñanza en sus diferentes niveles, etapas y tipos. Para conseguir esos objetivos, debería desarrollar acciones integradas en todos los poderes públicos del país que conduzcan a la extinción del analfabetismo, del absentismo escolar, la mejora de la calidad de la enseñanza, la formación para el trabajo, etc.

Las directrices para la educación nacional establecidas por este plan sirven para indicar el norte para las veinte metas planeadas para la próxima década (Pereira y Oliveira, 2014).

Además de las dos leyes de educación vigentes, se analizaron las directrices curriculares nacionales de los cursos de Pedagogía y licenciaturas. Las Directrices Curriculares Nacionales para el Curso de Pedagogía (DCN Pedagogía) están 
recogidas en el Parecer CNE/CP n. ${ }^{\circ}$, del 13 de diciembre de 2005, y en la Resolución CNE/CP n. ${ }^{\circ}$ 1, del 15 de mayo de 2006.

En el Parecer CNE/CP n. ${ }^{\circ}$ 5, del 13 de diciembre de 2005, se explicitan los fines, principios, objetivos, perfil del pedagogo, organización del curso de Pedagogía y la necesidad de aplicación de las directrices determinando sus procedimientos. La Resolución resultante de este Parecer establece efectivamente las Directrices Curriculares Nacionales para el Curso de Pedagogía.

Las Directrices Curriculares Nacionales para la Formación Inicial y Continua de los Profesionales del Magisterio de la Educación Básica (DCN Licenciaturas) comprenden el Parecer CNE/CP n. ${ }^{\circ}$ 2, del 9 de junio de 2015, y la Resolución n. ${ }^{\circ}$ 2, del 2 de julio de 2015.

El Parecer CNE/CP n. ${ }^{\circ}$ 2, del 9 de junio de 2015, expone la formación de los profesionales de la docencia en educación básica, explicando que esta formación constituye un campo de discusión sobre las concepciones, políticas, planes de estudio, importancia de la práctica para la formación profesional y valoración profesional.

Por su parte, la Resolución n. ${ }^{\circ}$ 2, del 2 de julio de 2015, establece las Directrices Curriculares Nacionales para la formación inicial en nivel superior y para la formación continua. Es preciso indicar que el 15 de abril de 2020 se publicaron las nuevas directrices curriculares nacionales para la formación inicial de profesores para la educación básica y la institución de la Base Nacional Común para la Formación (BNC). Estas Directrices cuentan con una moratoria de años para su ejecución obligatoria, por lo que en este momento no están siendo aplicadas y, por tanto, no pueden formar parte de los análisis de esta investigación.

\section{OBJETIVO DEL ARTí́CULO}

Mostrar la capacitación implícita o explícita ofrecida a los profesores en formación, sobre un aspecto primordial en la sociedad brasileña: la prevención del riesgo social partiendo del análisis de la legislación educativa brasileña.

\section{Metodología}

En la investigación que da origen a este artículo se ha utilizado metodología de tipo cualitativo por ser la modalidad de investigación que permite conocer directamente la realidad social, es decir, sin la mediación de definiciones conceptuales u operativas y de instrumentos de medida con alto grado de estructuración (Revuelta y Sánchez, 2009). El proceso metodológico seguido ha consistido en recoger información de fuentes documentales primarias y aplicarles un análisis de contenido como técnica de investigación (Ruiz, 2013; Mozzato y Grzybovski, 2011; Silva, Gobbi y Simão, 2005; Vallés, 2003; Krippendorff, 1997). 
MAGDA MARIA VENTURA GOMES DA SILVA

FORMACIÓN INICIAL DEL PROFESORADO BRASILEÑO EN PREVENCIÓN DEL RIESGO SOCIAL SEGÚN LA LEGISLACIÓN EDUCATIVA

El análisis de contenido es una técnica de investigación ampliamente utilizada (Ruiz, 2013), aunque su definición haya ido evolucionando desde enfoques centrados en la cuantificación de los resultados hasta enfoques centrados en la incorporación del dato cualitativo como la mayor riqueza de este procedimiento (Krippendorff, 1997). En el análisis de contenido se busca, a través de procedimientos sistemáticos y objetivos, la descripción de los contenidos de los mensajes con el objetivo de desvelar la información (Rozek, Serra y Brasil, 2014). "En esta interacción lingüística, tanto escrita como oral, se trata de encontrar el "contexto dentro del texto", o encontrar el sentido-significado» (Atehortúa y Zwerg-Villegas, 2012, p. 102).

En la investigación cualitativa, el investigador intenta encontrar unidades de contenido significativo para construir las categorías o subcategorías. Es indispensable «la construcción de un sistema categorial que organice la información y le dé sentido en el marco del proyecto» (Ballesteros y Mata, 2014, p. 34). Es decir, «el establecimiento de categorías constituye el núcleo central del análisis de contenido» (García, 2010, p. 164). Así, a través de esta metodología se han analizado todos los planes de estudio de la carrera docente de las dos últimas leyes brasileñas de educación y de las directrices curriculares nacionales de las licenciaturas y Pedagogía. De cada una de estas bases documentales se ha realizado un análisis cualitativo de contenido -utilizando el software Atlas.ti- fundamentado en la Grounded theory. "Teorias fundamentadas, por serem baseadas em dados, tendem a oferecer mais discernimento, melhorar o entendimento e fornecer um guia importante para a ação» (Strauss y Corbin, 2008, p. 25). Todo el análisis de contenido se ha realizado a través del software Atlas. ti.

El software Atlas.ti utiliza elementos importantes que permiten al investigador la construcción de un archivo para guardar y manejar todo el material necesario para trabajar (unidad hermenéutica), organizar los documentos primarios (los archivos de textos, imágenes y otros que se analizan) y las citas (los fragmentos de texto que se marcan en los documentos primarios por ser significativos para la investigación). Además de eso, permite construir los códigos (palabras clave que representan la unidad básica del análisis), los memos o anotaciones y las redes o representaciones gráficas de las relaciones entre códigos, familias, citas, etc. (Cuevas-Romo, Méndez-Valencia y Hernández-Sampieri, 2014).

En la investigación que da origen a este artículo, para realizar el análisis de contenido se ha procedido a ejecutar un preanálisis, la formación de un sistema de categorías, una codificación, un análisis y una interpretación. Los resultados del preanálisis han arrojado seis documentos primarios: dos leyes de educación publicadas en Brasil en los años 1996 y 2014 y cuatro directrices curriculares nacionales de los cursos de Pedagogía y licenciaturas.

Cada ley, resolución y parecer se ha dividido en varias unidades de análisis que contienen los títulos, antetítulos, capítulos, artículos y párrafos. La estrategia de inducción analítica seguida a través de distintas técnicas de codificación ha establecido el sistema de las categorías. Así, para el análisis e interpretación de la documentación se han efectuado dos niveles de análisis: el nivel manifiesto de la legislación educativa y el nivel latente de las informaciones. 
En el nivel manifiesto se analizaron las informaciones obtenidas en los títulos, antetítulos y otros apartados de las leyes y directrices curriculares de licenciaturas y Pedagogía, estableciendo una valoración cuantitativa de los datos obtenidos. En el nivel latente se ha realizado una valoración cualitativa de las informaciones, a partir de los contenidos de cada uno de los textos legales examinados. La fundamentación teórica para esta valoración cualitativa fue obtenida en los estudios sobre el análisis cualitativo de textos y en la aplicación de la codificación teórica de Strauss y Corbin (2008) y de Nortthcutt y McCoy (2004).

\section{RESUltados Y DisCUSIÓN}

La legislación de enseñanza puede entenderse como una herramienta de trabajo cuando se pretende conocer lo que es la educación nacional y cuáles son los reglamentos establecidos por las leyes de educación y sus documentos correspondientes para, a partir de ellos, realizar análisis críticos.

Sobre la formación de los profesores brasileños en prevención del riesgo social los documentos analizados se concentran en la fuente documental "planes de estudio". En ellos se han establecido las categorías y se han clasificado los códigos asociados a cada una de ellas.

Las categorías elegidas se refieren a diferentes términos relacionados principalmente con la formación y el desarrollo profesional y a conceptos asociados al riesgo social, así como los contenidos de los planes de estudio de Pedagogía y demás licenciaturas encontrados en las leyes de enseñanza. También se han establecido los códigos que representan las categorías y se han enumerado las citas. Resultado de este proceso ha sido la obtención de:

- 6 categorías emergentes

- 17 códigos asociados a estas categorías. (Véase en la Tabla 1 el Sistema de Categorías y Códigos Asociados en los Planes de estudio/Categorías emergentes).

El procedimiento de segmentación en unidades de análisis ha conducido a un total de:

- 516 citas textuales

- 10 memos para proporcionar el apoyo necesario a los análisis.

Para los 17 códigos se han obtenido un volumen total de:

- 409 citas asociadas y

- para los 10 memos, el total de 168 citas asociadas. 
MAGDA MARIA VENTURA GOMES DA SILVA

FORMACIÓN INICIAL DEL PROFESORADO BRASILEÑO EN PREVENCIÓN DEL RIESGO SOCIAL SEGÚN LA LEGISLACIÓN EDUCATIVA

En la Tabla 1 se recoge el Sistema de Categorías y Códigos Asociados en los planes de estudio, leyes de educación brasileñas y categorías emergentes y sus frecuencias.

\section{TABLA 1}

Sistema de Categorías y Códigos Asociados en los planes de estudio, leyes de educación brasileñas y categorías emergentes y sus frecuencias

\begin{tabular}{c|l|c|c}
\hline \multicolumn{1}{c|}{$\begin{array}{l}\text { Planes de estudio en las Leyes de Educación brasileñas } \\
\text { Categorías emergentes y códigos asociados }\end{array}$} & f & $\%$ \\
\hline 1 & $\begin{array}{l}\text { Formación y desarrollo profesional: } \\
\text { Competencias Docentes. Formación profesional. Valoración } \\
\text { profesional. }\end{array}$ & 133 & $32 \%$ \\
\hline 2 & $\begin{array}{l}\text { Planes de estudio Licenciaturas/Pedagogía: } \\
\text { Planes de estudio - contenidos. }\end{array}$ & 111 & $27 \%$ \\
\hline 3 & $\begin{array}{l}\text { Atención a los principios: } \\
\text { Derechos humanos y Ciudadanía. Educación inclusiva. Respeto a } \\
\text { la Diversidad. }\end{array}$ & 86 & $21 \%$ \\
\hline 4 & $\begin{array}{l}\text { Manifestaciones de riesgo social: } \\
\text { Discriminación. Drogas. Embarazo precoz. Estudiantes con } \\
\text { necesidades especiales. Jóvenes - en la calle, con libertad asistida } \\
\text { o privados de libertad. }\end{array}$ & 48 & $12 \%$ \\
\hline 5 & $\begin{array}{l}\text { Escolarización activa: } \\
\text { Apoyo y cooperación con familias. Búsqueda activa de niños y } \\
\text { adolescentes. }\end{array}$ & 19 & $5 \%$ \\
\hline 6 & $\begin{array}{l}\text { Riesgo social y conceptos relacionados: } \\
\text { Vulnerabilidad social. Violencia contra niños y adolescentes. } \\
\text { Exclusión. }\end{array}$ & 12 & $3 \%$ \\
\hline \begin{tabular}{l} 
Total \\
\hline
\end{tabular} & 409 \\
\hline
\end{tabular}

Fuente: Elaboración propia.

En el análisis del conjunto de las leyes educativas analizadas, las categorías emergentes más frecuentes fueron:

- la formación y desarrollo profesional (32\%),

- las informaciones sobre los contenidos de los planes de estudio licenciaturas/Pedagogía (27\%),

- la atención a los principios legales (21\%) y

- las manifestaciones de riesgo social (12\%). 
MAGDA MARIA VENTURA GOMES DA SILVA

FORMACIÓN INICIAL DEL PROFESORADO BRASILEÑO EN PREVENCIÓN DEL RIESGO SOCIAL SEGÚN LA LEGISLACIÓN EDUCATIVA

Los porcentajes más bajos los mostraron los términos relacionados con la escolarización activa ( $5 \%$ ) y con el riego social y sus conceptos asociados (3\%). Seguidamente se muestran las categorías emergentes, sus códigos asociados y la relación encontrada entre prevención del riesgo social y capacitación del profesorado en formación. Es preciso señalar que de todas estas categorías se eliminan, en este artículo, los aspectos que no guardan ninguna relación con el riesgo social, como por ejemplo la escolarización activa y algunos aspectos contemplados en otras categorías.

\subsection{Formación y desarrollo profesional}

En esta categoría se agrupan las informaciones sobre la formación profesional, la valoración profesional y las competencias docentes (véase el Diagrama 1). En estos códigos se muestran los conceptos y reglamentos establecidos por las leyes de enseñanza respecto a la formación inicial y desarrollo profesional.

DiAgrama 1

Red sobre la Formación y desarrollo profesional y códigos asociados

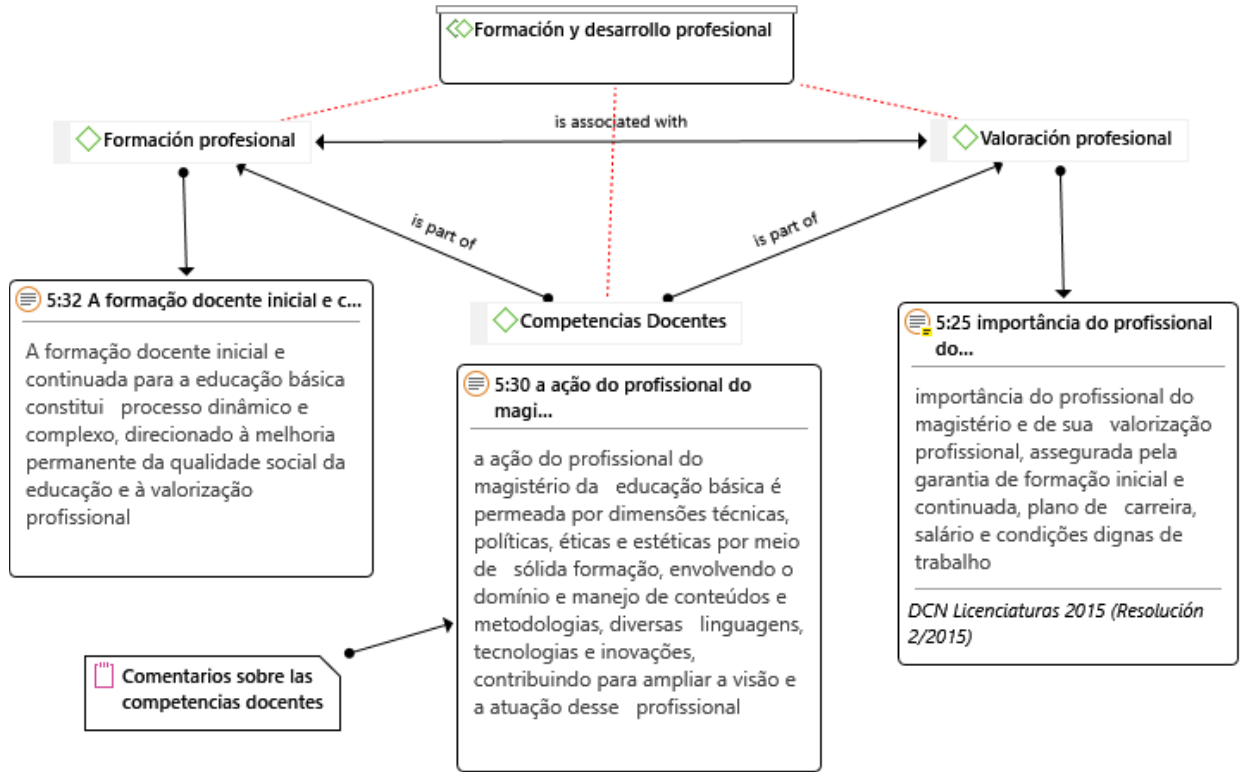

Fuente: Elaboración propia.

La formación de los profesionales de la docencia en educación básica constituye un campo de discusión que muestra la importancia de la teoría y práctica para la formación profesional inicial y continua de los profesores. Esta formación 
MAGDA MARIA VENTURA GOMES DA SILVA

FORMACIÓN INICIAL DEL PROFESORADO BRASILEÑO EN PREVENCIÓN DEL RIESGO SOCIAL SEGÚN LA LEGISLACIÓN EDUCATIVA

profesional está asociada a la valoración profesional, que incluye la articulación entre la formación inicial, continua, sueldos, carrera, remuneración y condiciones de trabajo. Las competencias docentes son parte de esta formación y valoración profesional. Todos los documentos analizados hacen referencia a la formación y valoración profesional.

Sobre la formación profesional, en la LDB se establecen los fundamentos de la formación y los planes de estudio de los cursos de formación. La base de referencia es la Base Nacional Común Curricular. En el PNE las metas sobre la formación de los profesionales de la educación muestran que el objetivo es garantizar la política nacional de formación de estos profesionales, de acuerdo con la LDB, en régimen de colaboración entre el Gobierno Nacional, los Estados, el Distrito Federal y los Municipios.

De entre todas las metas del PNE, con respecto a la formación en prevención del riesgo social se destaca la meta 7 (estrategia 7. 23): "Garantizar las políticas contra la violencia en la escuela, incluyendo el desarrollo de acciones dirigidas a la formación de educadores para detectar las señales de sus causas, como la violencia doméstica y sexual» (10:97 - D10: PNE 2014.rtf). A través de esta meta, se identifica una preocupación explícita con las políticas contra la violencia escolar y acciones para la capacitación docente.

Centrándose en las directrices curriculares nacionales en la formación del profesorado para la Educación Básica (DCN Licenciaturas), esta formación debe contribuir a consolidar una nación democrática, justa e inclusiva, reconociendo y valorando "la diversidad y, por lo tanto, contraria a toda forma de discriminación" (4:59 - D4: DCN 2015 Licenciaturas Parecer 2.pdf). Así se identifica, a través de una visión general, la preocupación explícita en un país que debe realizar la inclusión y reconocer y valorar la diversidad. Es decir, un país contrario a la discriminación.

La valoración de la docencia se entiende como una dimensión constitutiva y constituyente de su formación inicial y continua. Todos los documentos analizados identificaron esta manera de expresar la valoración.

Las competencias docentes solo se muestran en las directrices curriculares de Pedagogía y licenciaturas a través de textos similares. En ellos, no se usa explícitamente el término "competencia». Este término se deduce al mostrar lo que se espera de los estudiantes que concluyen su formación. Ese es el procedimiento implícito para identificar las competencias docentes. En la formación del pedagogo y de los demás licenciados se muestran ejemplos de lo que se espera de ellos al concluir los estudios (véase el Cuadro 2). 
CUADRO 2

Directrices curriculares nacionales: ejemplos de lo que se espera de los estudiantes que concluyen la formación en las licenciaturas y Pedagogía

Ejemplos de lo que se espera de los estudiantes que concluyen la formación en las licenciaturas y Pedagogía (Directrices curriculares nacionales - Brasil)

- Identificar problemas socioculturales y educacionales, con el objetivo de contribuir a la superación de exclusiones sociales, étnico-raciales, económicas, culturales, religiosas y políticas.

- Demostrar conciencia de la diversidad, respetando las diferencias.

- Realizar investigaciones sobre los estudiantes y la realidad sociocultural en la que ellos viven sus experiencias.

Fuente: Elaboración propia.

Como resumen de los análisis realizados a la categoría "Formación y desarrollo profesional», no se ha identificado información directamente relacionada con la capacitación del profesorado en prevención del riesgo social. Sin embargo, se indica que la meta 7 del PNE expresa una preocupación explícita con las políticas contra la violencia escolar y la realización de acciones para la capacitación docente.

Otros textos legales mostraron su preocupación por:

- desarrollar valores éticos y ciudadanos,

- conciencia de la diversidad,

- respeto a las diferencias,

- lucha contra la discriminación y exclusión social.

En estos ejemplos se puede identificar de manera implícita la preocupación por situaciones de riesgo social, pero sin relacionarse con la capacitación docente en la prevención.

\subsection{Planes de estudio licenciaturas/Pedagogía}

Esta categoría agrupa los contenidos de los «Planes de estudio/contenidos" con 111 referencias (citas), identificadas en todos los documentos. En la LDB se muestra la información sobre «el contenido que trate de los derechos de los niños y de los adolescentes, teniendo como directriz la Ley n. ${ }^{\circ} 8.069$, del 13 de julio de 1990, que instituye el Estatuto del Niño y Adolescente» (9:26 - D9: LDB 1996.rtf). Además de eso, en el artículo 25 § 9. ${ }^{\circ}$ de la ley se afirma que la prevención de todos los tipos de violencia contra los niños y adolescentes deberá ser estudiada como tema transversal en los planes de estudio. Sin embargo, la prevención no representa contenido obligatorio. 
MAGDA MARIA VENTURA GOMES DA SILVA

FORMACIÓN INICIAL DEL PROFESORADO BRASILEÑO EN PREVENCIÓN DEL RIESGO SOCIAL SEGÚN LA LEGISLACIÓN EDUCATIVA

La ley, en la educación básica, muestra los contenidos para los planes de estudio relacionados con la diversidad de razas en la formación histórica y cultural brasileña, la base común nacional y una parte diversificada:

- respetar las características regionales y locales de la sociedad,

- respetar las características de la cultura,

- respetar las características de la economía del país,

- respetar las características de los estudiantes.

El PNE muestra la necesidad de incentivar la inclusión en los cursos de licenciatura de procesos de enseñanza-aprendizaje relacionados con "la atención educativa de alumnos con discapacidad, trastornos globales del desarrollo y altas habilidades o superdotación» (10:26 - D10: PNE 2014.rtf). Para eso, se debe combinar "formación general y específica con la práctica didáctica, además de la educación para las relaciones étnico-raciales, la diversidad y las necesidades de las personas con discapacidad" (10:34 - D10: PNE 2014.rtf).

En las DCN Pedagogía se muestran tres núcleos de estudios constitutivos de los planes: núcleo de estudios básicos, núcleo de profundización y diversificación de estudios y núcleo de estudios de integración.

Sobre ellos se destacan los contenidos relacionados con el objeto de la investigación (Cuadro 3). En las DCN Licenciaturas los tres núcleos de estudios componentes de los planes se muestran de manera similar a los núcleos de las DCN Pedagogía.

\section{CUADRO 3}

DCN Pedagogía: Núcleos de estudios y ejemplos de contenidos para los planes de estudios

\begin{tabular}{c|l}
\hline $\begin{array}{c}\text { Núcleo de } \\
\text { estudios básicos }\end{array}$ & $\begin{array}{l}\text { «Estudio de las relaciones entre educación y trabajo, diversidad } \\
\text { cultural, ciudadanía, sostenibilidad, entre otras problemáticas } \\
\text { centrales de la sociedad contemporánea» (8:29 - D8: DCN Pedagogía } \\
\text { 2006 RES 1.pdf.) }\end{array}$ \\
\cline { 2 - 2 } & $\begin{array}{c}\text { "La importancia de conocer las políticas de educación inclusiva } \\
\text { y comprender sus implicaciones organizacionales y pedagógicas, } \\
\text { para la democratización de la Educación Básica en el país» (6:56 - } \\
\text { D6: DCN Pedagogia 2005 Parecer 5.pdf). }\end{array}$ \\
\hline $\begin{array}{c}\text { Núcleo de } \\
\text { Profundización } \\
\text { yiversificación } \\
\text { de los estudios }\end{array}$ & $\begin{array}{l}\text { "Evaluación, creación y uso de textos, materiales didácticos, } \\
\text { procedimientos y procesos de aprendizaje que contemplen la } \\
\text { diversidad social y cultural de la sociedad brasileña» (8:35 - D8: } \\
\text { DCN Pedagogía 2006 RES 1.pdf). }\end{array}$ \\
\hline
\end{tabular}

Fuente: Elaboración propia. 


\subsection{Atención a los principios}

Esta categoría comprende el respeto a la diversidad, los derechos humanos y ciudadanía y la educación inclusiva como temas más frecuentes en la representación de los principios relacionados con el riesgo social (véase el Diagrama 2).

DiAGRAMA 2

Atención a los principios y sus códigos asociados

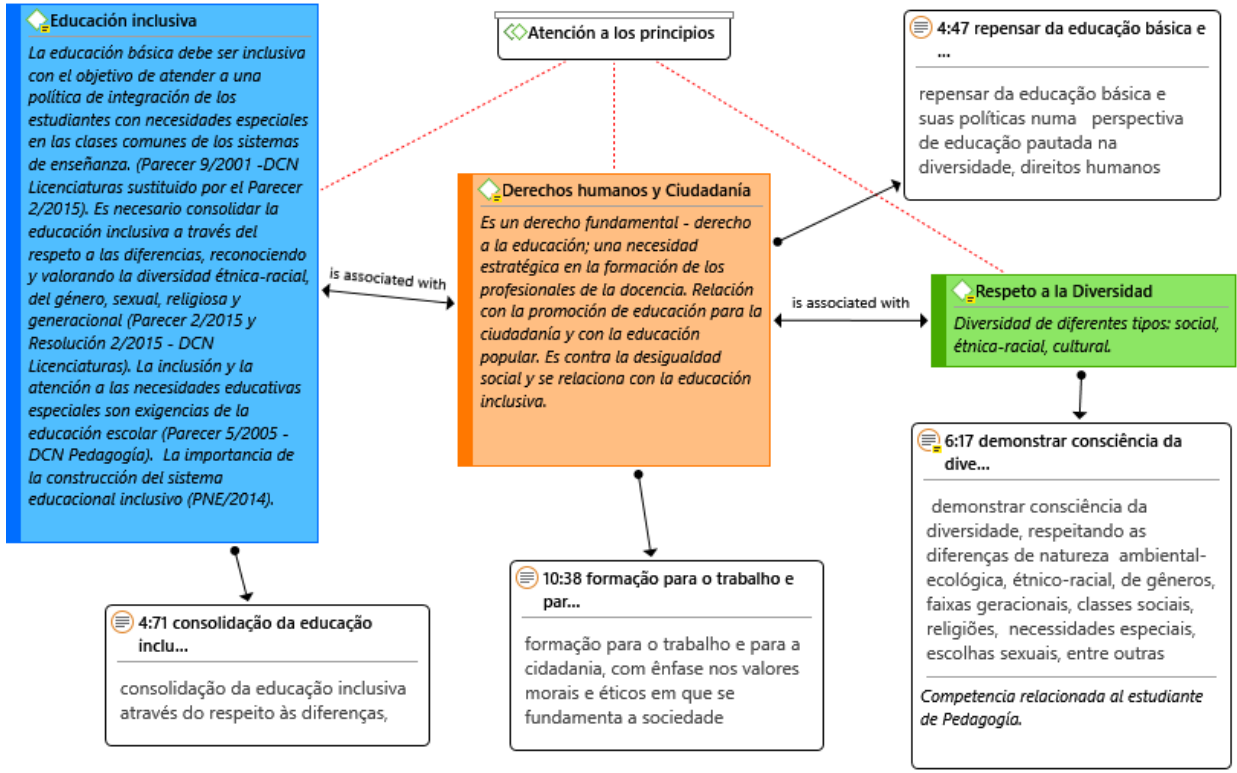

Fuente: Elaboración propia.

El respeto a la diversidad social, étnico-racial, cultural, de género, sexual, religiosa, los derechos humanos y la ciudadanía se muestra en todos los documentos analizados.

En cuanto al término educación inclusiva se presenta en todos los documentos, excepto en la LDB. Para las DCN Pedagogía la inclusión representa un principio del trabajo educativo y una exigencia de la educación escolar. Consecuentemente, los profesores deberán sentirse siempre dispuestos a trabajar con postura ética y profesional. En las DCN de las licenciaturas lo que se muestra es la necesidad de consolidar la educación inclusiva a través del respeto a las diferencias y de políticas de inclusión puesto que la escuela brasileña debe ser inclusiva. 
MAGDA MARIA VENTURA GOMES DA SILVA

FORMACIÓN INICIAL DEL PROFESORADO BRASILEÑO EN PREVENCIÓN DEL RIESGO SOCIAL SEGÚN LA LEGISLACIÓN EDUCATIVA

\subsection{Manifestaciones de riesgo social}

Esta categoría se refiere a los términos usados en las leyes que identifican las siguientes manifestaciones: estudiantes con necesidades especiales, discriminación, jóvenes de la calle, jóvenes en libertad asistida o privados de libertad, drogas y embarazo precoz. Sin embargo, la legislación no identifica estos términos con la expresión "manifestaciones de riesgo social».

Los estudiantes con necesidades especiales son los que poseen «discapacidades y trastornos globales del desarrollo y altas habilidades o superdotación». Sobre ellos se han encontrado referencias en todos los documentos, principalmente en la LDB y en el PNE.

La LDB explica la atención especializada a estos estudiantes realizada por los sistemas de enseñanza y el poder público. Por primera vez una ley de educación señala la educación especial como deber constitucional, así como la formación y capacitación de los profesores para atender a estos estudiantes. En el PNE se destacan las informaciones sobre la creación de centros multidisciplinares, integrados por profesionales de salud, asistencia social, pedagogía y psicología, para apoyar el trabajo de los profesores.

Sobre la discriminación o prejuicio se encuentran referencias en el PNE y en las DCN Licenciaturas. Los dos documentos identifican las mismas expresiones: erradicación de la discriminación e implementación de políticas de prevención a la evasión motivada por cualquier forma de discriminación.

En cuanto a los jóvenes de la calle, con libertad asistida o privados de libertad se encuentran referencias también en el PNE y en las DCN Licenciaturas. Los términos usados son: adolescentes y jóvenes en régimen de libertad asistida; en cumplimiento de medidas socioeducativas; en situación de calle, y personas privadas de libertad.

Sobre drogas y embarazo precoz se encuentra información solo en el PNE, que determina hacer el acompañamiento de las situaciones del consumo de drogas y embarazo precoz con la colaboración de las familias y con los órganos públicos de asistencia social, salud y protección a la adolescencia y juventud.

La red elaborada sobre las manifestaciones de riesgo social en las leyes de educación resume las informaciones de sus componentes. A la vez, representa los vínculos que se establecen entre ellos (véase el Diagrama 3). 
DIAGRAMA 3

Manifestaciones de riesgo social - leyes de enseñanza: vínculos y frases de efecto

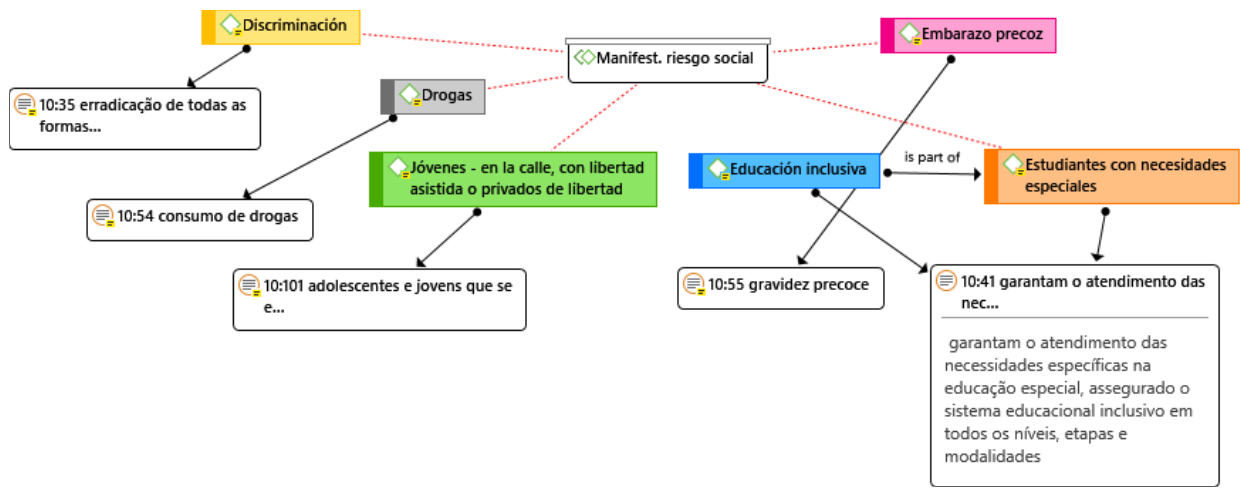

Fuente: Elaboración propia.

\subsection{Riesgo social y conceptos relacionados}

Esta categoría comprende los conceptos de vulnerabilidad social; violencia contra niños y adolescentes y exclusión (véase el diagrama 4). En torno a la vulnerabilidad social se ha identificado solo una información en el PNE (Meta 6, Estrategia 6.2) sobre el programa de construcción de escuelas en las comunidades pobres y con niños en situación de vulnerabilidad social.

Por primera vez en el plan de educación se recala sobre el concepto de vulnerabilidad social, aunque sea de forma superficial puesto que no se señala nada diferente a la construcción de escuelas. No se hace ninguna mención a la necesidad de capacitar a los docentes para la prevención.

Centrándose en la violencia contra niños y adolescentes solo se presenta en las dos leyes de enseñanza (LDB y PNE). En la LDB se muestra la "prevención de todas las formas de violencia contra el niño y el adolescente» (9:1 - D9: LDB 1996. rtf) y en el PNE «el combate a las situaciones de discriminación, prejuicio y violencia» (10:5 - D10: PNE 2014.rtf).

Sobre el término Exclusión se encuentran referencias en los documentos analizados excepto en la LDB. En la Meta 6 del PNE se muestra la necesidad de una "red de protección contra las formas asociadas de exclusión» (10:58 - D10: PNE 2014.rtf). Esta es una estrategia que limita la política de prevención al abandono escolar por prejuicio u otros tipos de discriminación (Estrategia 3.13). Así, se ha encontrado una preocupación explícita hacia la prevención del riesgo social, lo que lleva a identificar, de manera implícita, la necesidad de capacitar a los profesores en su prevención.

En las DCN Pedagogía y Licenciaturas se identifica el mismo texto: "Contribuir a la superación de las exclusiones sociales, étnico-raciales, económicas, culturales, 
MAGDA MARIA VENTURA GOMES DA SILVA

FORMACIÓN INICIAL DEL PROFESORADO BRASILEÑO EN PREVENCIÓN DEL RIESGO SOCIAL

SEGÚN LA LEGISLACIÓN EDUCATIVA

religiosas, políticas, de género, sexuales y otras» (5:16 - D5: DCN 2015 Licenciaturas RES 2.pdf). Como puede observarse existe la preocupación por las manifestaciones de riesgo social, pero falta establecer la relación explícita con la capacitación del profesorado, en formación, para prevenir las manifestaciones de riesgo social.

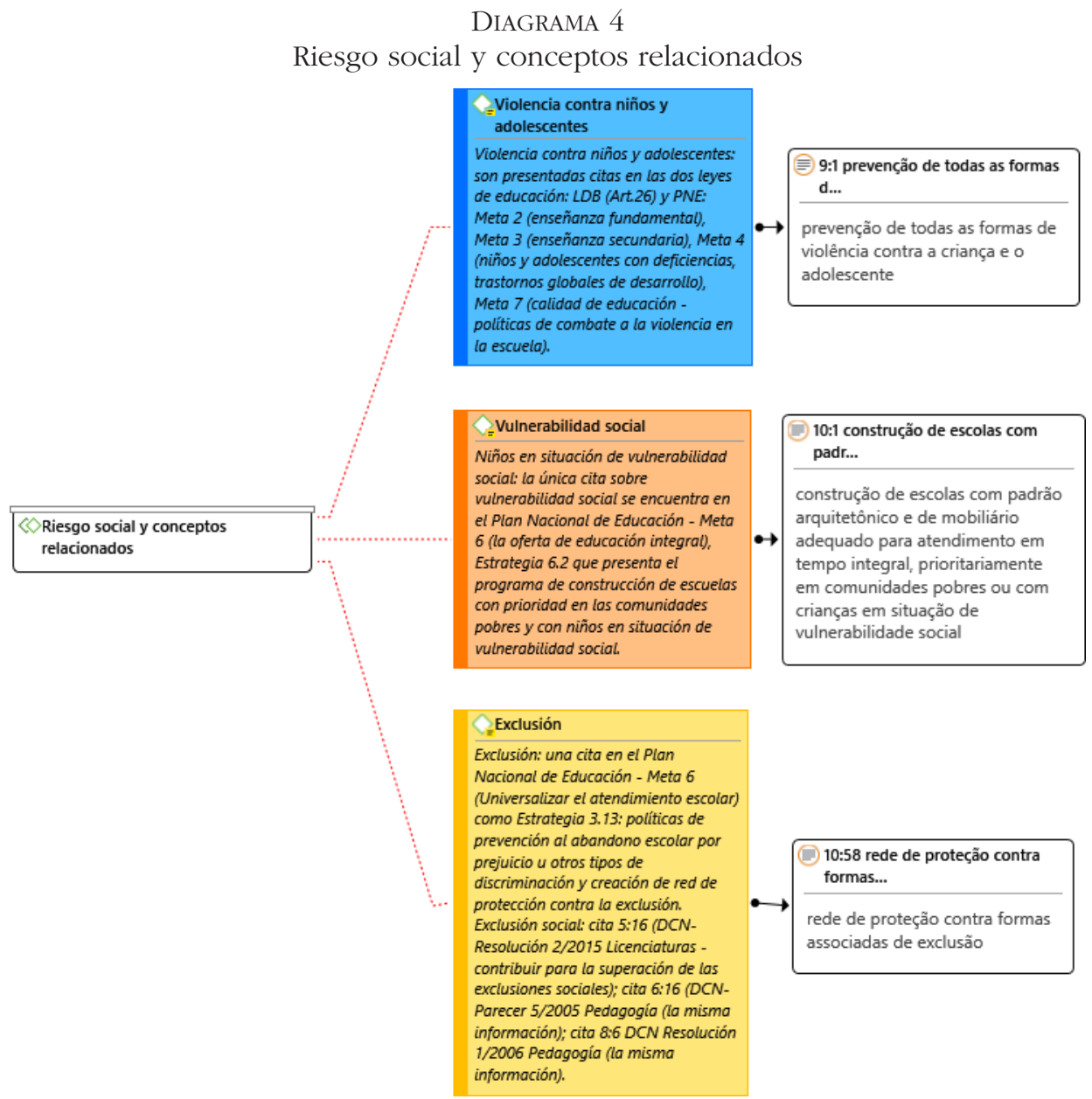

Fuente: Elaboración propia.

\section{CONCLUSIÓN}

El análisis de contenido realizado a la legislación educativa brasileña ha perseguido el objetivo de encontrar, de manera implícita o explícita, la capacitación 
ofrecida a los profesores en formación sobre un aspecto primordial en la sociedad brasileña: la prevención del riesgo social.

Como conclusiones del análisis realizado puede indicarse que, aunque en las leyes de educación se señale que hay que atender las peculiaridades que ocurren en la sociedad, esto es, las desigualdades, la diversidad, la discriminación, las drogas, los niños y jóvenes de la calle, la vulnerabilidad social, la violación de los derechos humanos, en definitiva, todos los problemas de la sociedad brasileña que pueden propiciar el nacimiento del riesgo social, en los documentos analizados no aparecen indicaciones ni se desarrollan apartados que concreten ese objetivo.

Fortalece esta primera conclusión el hecho de que en los documentos sometidos a análisis de contenido no se incide en la formación del profesorado en ningún aspecto que les capacite para poder prevenir el riesgo social desde la escuela. Así, los profesores llegan a las escuelas sin ninguna preparación, por lo que en ella solo pueden padecer los graves problemas que origina el riesgo social en sus clases, escuelas y en comunidades.

A pesar de haber en el Plan Nacional de Educación, de manera implícita, una gran preocupación por abordar la violencia escolar y manifestar, también de manera implícita, la necesidad de desarrollar acciones dirigidas a la formación de educadores para detectar las señales de violencia, en este documento solo se mostró esta idea en un párrafo de la ley.

Desafortunadamente, las políticas educativas desarrolladas en las leyes y en las directrices curriculares nacionales solo se focalizan en transmitir conocimientos a los docentes en formación. Este hecho representa una contradicción entre las necesidades de la sociedad brasileña y las teorías academicistas totalmente encerradas en los despachos ministeriales.

La sociedad brasileña necesita y demanda a las leyes educativas capacitar al profesorado en prevención del riesgo social más que en conocimientos academicistas durante su formación inicial.

En resumen, por todo lo expuesto en el artículo «Formación inicial del profesorado brasileño en prevención del riesgo social según la legislación educativa", se concluye afirmando que la competencia prevención del riesgo social no está entre las competencias de formación del profesorado brasileño, un país caracterizado por ser violento, desigual, por tener altas tasas de criminalidad, ausencia de servicios básicos, problemas de vivienda y de saneamiento, niños y adolescentes viviendo en las calles, altas tasas de trabajo infantil de riesgo, lugares habitacionales insalubres o favelas, violencia en las escuelas y en las comunidades y muchas otras manifestaciones de riesgo social que resultan invisibles para el Estado y la legislación educativa. 
MAGDA MARIA VENTURA GOMES DA SILVA

FORMACIÓN INICIAL DEL PROFESORADO BRASILEÑO EN PREVENCIÓN DEL RIESGO SOCIAL SEGÚN LA LEGISLACIÓN EDUCATIVA

\section{REFERENCIAS BIBLIOGRÁFICAS}

Almeida, A., Lisboa, C. y Caurcel, M. J. (2007). ¿Por qué ocurren los malos tratos entre iguales? Explicaciones causales de adolescentes portugueses y brasileños. Revista Interamericana de Psicología/Interamerican Journal of Psychology, 41 (2), 107-118.

Atehortúa, F. H. R. y Zwerg-Villegas, A. M. (2012). Metodología de la investigación: más que una receta. AD-minister, Medellín-Colombia, 20, enero-junio, 91-111.

Ballesteros, V. y Mata, B. (2014). Sentido y forma de la investigación cualitativa. En V. Ballesteros (Coord.), Ciencias Sociales y Jurídicas. Taller de Investigación Cualitativa (pp. 11-46). Madrid: UNED. Edición digital.

Constituição da República Federativa do Brasil. (Const.). (1988). Brasília, DF: Senado Federal, Centro Gráfico. Recuperado de: http://www.planalto.gov.br/ccivil_03/Constituicao/Constituicao.htm.

Cuevas-Romo, A., Méndez-Valencia, S. y Hernández-Sampieri, R. (2014). Manual de introducción a ATLAS.ti 7. Universidad de Celaya.

García, J. L. (2010). Métodos de investigación en educación: Investigación cualitativa y evaluativa, vol. II (2. ${ }^{a}$ reimpresión). Madrid: UNED..

Gonçalves, R. S. (2013). Favelas do Rio de Janeiro: História e direito. Rio de Janeiro: Pallas - Ed. PUC-Rio.

IBGE. (2019). Síntese de indicadores sociais: uma análise das condições de vida da população brasileira. IBGE, Coordenação de População e Indicadores Sociais. Rio de Janeiro: IBGE. Recuperado de biblioteca.ibge.gov.br.

IBGE. (2020). Estimativas da população residente no Brasil e unidades da federação com data e referência em $1 .^{\circ}$ de julho de 2020. IBGE, Diretoria de Pesquisas. Coordenação de População e Indicadores Sociais. Rio de Janeiro: IBGE. Recuperado de ftp.ibge.gov.br.

Janczura, R. (2012). Risco ou vulnerabilidade social? Textos \& Contextos, Porto Alegre, II (2), 301-308.

Krippendorff, K. (1997). Metodología de análisis de contenido. Teoría y práctica. Barcelona: Paidós Comunicación.

Lebrero, M. P. y Quicios, M. P. (2011). La desprotección invisible, nueva raíz del riesgo social infantil y juvenil. Pedagogía Social. Revista Interuniversitaria, 18, Sevilla, España: Sociedad Iberoamericana de Pedagogía Social.

Ley n. ${ }^{\circ}$ 13.415, del 16 de febrero de 2017. Modifica la Ley n. ${ }^{\circ}$ 9.394, del 20 de diciembre de 1996, que establece las Directrices y Bases de la Educación Nacional y 11.494, del 20 de junio de 2007, que reglamenta el Fondo de Mantenimiento y Desarrollo de la Educación Básica y de Valoración de los Profesionales de la Educación, la Consolidación de las Leyes del Trabajo - CLT... Brasília, Brasil. Diário Oficial da União, del 17 de febrero de 2017.

Ley n. ${ }^{\circ} 13.005$, del 25 de junio de 2014. Aprueba el Plan Nacional de Educación - PNE y establece otras providencias. Brasília, Brasil. Diário Oficial da União, 26 de junio de 2014.

Ley n. ${ }^{\circ}$ 9.394, del 20 de diciembre de 1996. Establece las Directrices y Bases de la Educación Nacional. Brasília, Brasil. Diário Oficial da União, 24 de diciembre de 1996.

Ley n. ${ }^{\circ}$ 8.069, del 13 de julio de 1990. Instituye el Estatuto del Niño y Adolescente y establece otras providencias. Brasília, Brasil. Diário Oficial da República Federativa do Brasil, 16 de julio de 1990.

Lorence, B. (2008). Procesos de socialización parental con adolescentes de familias en situación de riesgo psicosocial. Madrid: Documentos de Trabajo - Fundación Acción Familiar. 
MAGDA MARIA VENTURA GOMES DA SILVA

FORMACIÓN INICIAL DEL PROFESORADO BRASILEÑO EN PREVENCIÓN DEL RIESGO SOCIAL SEGÚN LA LEGISLACIÓN EDUCATIVA

Mozzato, A. R. y Grzybovski, D. (2011). Análise de conteúdo como técnica de análise de dados qualitativos no campo da administração: potencial e desafios. Rev. Adm. Contemp., 15 (4), 731-747.

Northcutt, N. y McCoy, D. (2004). Interactive Cualitative Análisis. A sistem method for qualitative research. California: Sage publications.

Parecer CNE/CP n. ${ }^{\circ}$ 2, del 9 de junio de 2015. Directrices Curriculares Nacionales para la Formación Inicial y Continua de los Profesionales del Magisterio de la Educación Básica. Brasília, Brasil. DOU, 25/6/2015, Sección 1, p. 13.

Parecer CNE/CP n. ${ }^{\circ}$ 5, del 13 de diciembre de 2005. Establece las Directrices Curriculares Nacionales para el Curso de Graduación en Pedagogía, licenciatura. Brasília, Brasil. Diário Oficial da União, 15/5/2006.

Pereira, A. M. y Minasi, L. F. (2014). Um Panorama Histórico da Política de Formação de Professores no Brasil. Revista de Ciências Humanas, 15 (24), 7-19.

Pereira, T. V. y Oliveira, V. B. (2014). Base Nacional Comum: a Autonomia Docente e o Currículo Único em Debate. Revista Teias, 15 (39), 24-42.

Quicios, M. P. (2007). Prevención del riesgo social a través de una docencia competente. Acción Pedagógica, 16 (enero-diciembre), 144-153.

Quicios, M. P. y Triguero, J. (Dirs.). (2012). La comunidad educativa previene la delincuencia juvenil. Londres: Pearson.

Quicios, M. P. y Velloso, A. (2012). Delincuencia juvenil. En M. P. Quicios y J. Triguero (Dirs.). (2012). La comunidad educativa previene la delincuencia juvenil (pp. 21-40). Londres: Pearson.

Resolución CNE/CP n. ${ }^{\circ}$ 1, del 15 de mayo de 2006. Establece las Directrices Curriculares Nacionales para el Curso de Graduación en Pedagogía, licenciatura. Brasília, Brasil. Diário Oficial da União, 16 de mayo de 2006, Sección 1, p. 11.

Resolución CNE/CP n. ${ }^{\circ}$ 2, del 2 de julio de 2015. Establece las Directrices Curriculares Nacionales para la formación inicial en nivel superior (cursos de licenciaturas, cursos de formación pedagógica para los graduados y cursos de segunda licenciatura) y para la formación continua. Brasília, Brasil. Diário Oficial da União, 2 de julio de 2015, Sección 1, pp. 8-12. Rectificación publicada en $D O U, 3$ de julio de 2015, Sección 1, p. 28.

Revuelta, F. I. D. y Sánchez Gómez, M. C. (2009). Programas de análisis cualitativo para la investigación en espacios virtuales de formación. Recuperado de http://campus.usal. es/ teoriaeducacion/rev_numero_04/n4_art_revuelta_sanchez.htm

Rizzini, I. y Brasil do Couto, R. M. (2019). Children and adolescents on the streets: Main research themes in Brazil. Civitas-Revista de Ciências Sociais, 19 (1), 105-122.

Rodríguez, J. J. (2013). Infancia, adolescencia y juventud en dificultad social. En M. P. Quicios (Coord.), Infancia, adolescencia y juventud en dificultad social (pp. 1-28). Madrid: McGraw-Hill.

Rozek, M., Serra, R. y Brasil, P. (2014). As dificuldades de aprendizagem e o processo de escolarização: Uma perspectiva de investigação qualitativa na educação. Atas CIAIQ 2014. 3. ${ }^{\circ}$ Congesso Iberoamericano de Investigação Qualitativa.

Ruiz, I. C. (2013). El análisis de contenido en la investigación educativa: propuesta de fases y procedimientos para la etapa de evaluación de la información. Pedagogía Universitaria, 14 (3), 71-93.

Sampó, C. (2018). Brasil: la re-significación de la violencia como resultado del avance de organizaciones criminales. Revista de Estudios en Seguridad Internacional, 4 (1), 127146. 
MAGDA MARIA VENTURA GOMES DA SILVA

FORMACIÓN INICIAL DEL PROFESORADO BRASILEÑO EN PREVENCIÓN DEL RIESGO SOCIAL SEGÚN LA LEGISLACIÓN EDUCATIVA

Santos, A. R. (2007). LDB 9.394/96: Alguns passos na formação de professores no Brasil. En M. A. Granville (Org.). (2007). Teorias e práticas na formação de professores. Campinas, SP: Papirus, 17-30.

Santos, N. L., Roesch, D. y Cruz, L. R. (2014). Vulnerabilidade e risco social: produção de sentidos no campo socioassistencial. Revista Jovens Pesquisadores, 4 (1), 119-127.

Saviani, D. (2009). Formação de professores: aspectos históricos e teóricos do problema no contexto brasileiro. Revista Brasileira de Educação, 40 (14), 143-155.

Schwonke, C. R. G. B., Fonseca, A. D. y Gomes, V. L. (2009). Vulnerabilidades de adolescentes com vivencias de rua. Escola Anna Nery, 13 (4), 849-855. https://doi.org/10.1590/ S1414-81452009000400022

Silva, C. R., Gobbi, B. C. y Simão, A. A. (2005). O uso da análise de conteúdo como uma ferramenta para a pesquisa qualitativa: descrição e aplicação do método. Organizações Rurais \& Agroindustriais, Lavras, 7 (1), 70-81.

Strauss, A. L. y Corbin, J. (2008). Pesquisa Qualitativa: Técnicas e procedimentos para o desenvolvimento de teoria fundamentada (2. ${ }^{a}$ ed.). Tradução: Luciane de Oliveira da Rocha. Porto Alegre: Artmed.

Vallés, M. S. (Ed.). (2003). Técnicas cualitativas de investigación social: Reflexión metodológica y práctica profesional. Madrid: Síntesis.

\section{REFERENCIAS ATLASTI}

4:59 -... la diversidad y, por lo tanto... (23:879 [23:1266]) - D4: DCN 2015 Licenciaturas Parecer 2.pdf

5:16 - contribuir a la superación de... (8:861 [8:1001]) - D5: DCN 2015 Licenciaturas RES 2.pdf

6:56 - la importancia de conocer las políticas... (12:3368 [12:3563]) - D6: DCN Pedagogía 2005 Parecer 5.pdf

8:29 - estudio de las relaciones entre educación (3:3457 [3:3618]) - D8: DCN Pedagogía 2006 RES 1.pdf

8:35 - evaluación, creación y uso de (4:740 [4:910]) - D8: DCN Pedagogía 2006 RES 1.pdf

9:1 - prevención de todas las formas de... (22996:23069) - D9: LDB 1996.rtf

9:26 - el contenido que trate de los derechos... (28952:29127) - D9: LDB 1996.rtf

10:5 - el combate a las situaciones de discriminación... (29651:29796) - D10: PNE 2014.rtf

10:26 - la atención educativa de alumnos con discapacidad... (32209:32660) - D10: PNE 2014.rtf

10:34 - combinando formación general y... (71949:72239) - D10: PNE 2014.rtf

10:58 - red de protección contra las formas... (25258:25310) - D10: PNE 2014.rtf

10:97 - garantizar las políticas contra... (47577:47747) - D10: PNE 2014.rtf 
